\title{
LEGALIZACIÓN DE LA MARIHUANA EN CHILE: UN TEMA DE REFLEXIÓN PARA ENFERMERÍA
}

\author{
LEGALIZATION OF MARIJUANA IN CHILE: \\ A TOPIC FOR REFLECTION FOR NURSING
}

\author{
Carolina Luengo Martínez \\ Patricia Jara Concha ${ }^{* *}$
}

\begin{abstract}
RESUMEN
El artículo tiene como objetivos describir el contexto de la regularización de la marihuana en Chile y proporcionar algunas directrices con miras a que los profesionales de enfermería comiencen una reflexión al respecto. El profesional de enfermería tiene una gran responsabilidad en contribuir a mejorar la salud de la población, por lo que no puede restarse de este tema contingente. Se plantea que a lo menos cinco directrices debiesen ser consideradas para comenzar una reflexión: la mejor evidencia científica disponible, las posturas que han surgido al respecto, los desafíos que trae el cuidar en un mundo global, el cuidado cultural y finalmente los aspectos bioéticos involucrados. Y que enfermería debe enfatizar sus esfuerzos en la prevención del uso de la marihuana en etapas tempranas de la vida escolar.
\end{abstract}

Palabras clave: Cannabis, abuso de drogas, enfermería, globalización.

\begin{abstract}
The article aims to describe the context of the legalization of marijuana in Chile and provide some guidelines for nurses to begin reflecting about the implications it has. Nurses are commited to helping improve the health of the population; therefore, they cannot avoid being involved in the discussion around this issue. At least five guidelines are suggested to be considered to start a reflection: the best scientific evidence available, the views that have emerged about this issue, the challenges regarding care in a globalized world, cultural care and finally, the bioethical aspects involved. Nurses should focus their efforts in preventing marijuana use in early school years.
\end{abstract}

Key words: Cannabis, drug abuse, nursing, globalization.

Fecha recepción: 05/11/14 Fecha aceptación: 23/10/15

\footnotetext{
* Enfermera, Doctor (c) en Enfermería, Magíster en Salud Pública. Académica Universidad del Bío Bío, Chillán, Chile. Email: caroluengo@gmail.com

** Enfermera, Doctora en Enfermería, Profesora Titular Facultad de Enfermería, Universidad de Concepción, Concepción, Chile.E-mail: pjara@udec.cl
} 


\section{INTRODUCCIÓN}

La globalización ha causado un impacto en la salud de las poblaciones, imponiendo nuevos desafíos sanitarios a nivel mundial, en gran parte debido a la transferencia internacional de riesgos y oportunidades en salud, que ha generado una serie de peligros emergentes (1), tales como el abuso de drogas, ya que no solamente las personas y los microorganismos patógenos viajan de un país a otro; también lo hacen las ideas y los estilos de vida (1). En este mismo contexto global, los avances tecnológicos han posibilitado la mejoría en las condiciones de vida de la población, pero también han facilitado otros aspectos que dificultan la preservación de la salud, como el incremento constante de las adicciones, reflejo de la mayor producción de drogas en diferentes regiones del mundo y de su distribución indiscriminada, que como describen García y Ramírez ha "permeado todos los espacios sociales" (2).

Se esta frente a un fenómeno complejo, de carácter internacional que ha concertado un trabajo mancomunado entre diferentes actores sociales: públicos, privados, del área política, económica, religiosa, de salud, en búsqueda de lineamientos para una toma de decisión más efectiva acerca de este problema, del cual enfermería no puede estar ausente, requiriéndose de su activa participación como parte de un equipo interdisciplinario (2). Considerando que las/os enfermeras/os son los profesionales de salud más cercanos a las personas, tienen una gran responsabilidad en contribuir a mejorar la salud de la población.

Sin embargo, en este último tiempo este fenómeno de las adicciones y las drogas se ha complejizado aún más, tal es el caso de la marihuana, ya que han surgido alrededor del mundo varias iniciativas para su legalización, creando condiciones legales para su acceso, lo que ha generado diversas posiciones en favor y en contra.
Por tanto, es oportuno y contingente que las enfermeras y enfermeros comiencen a realizar un análisis del tema; es así como el propósito de este documento, por un lado, es describir el contexto de la regularización de la marihuana en Chile y, por otro, proporcionar algunas directrices que debiesen ser consideradas para que los profesionales de enfermería comiencen una reflexión del tema con miras en aunar criterios referentes a un enfoque en común que favorezca los cuidados otorgados a la persona, familia y comunidad.

\section{DESARROLLO}

\section{El consumo de marihuana: un problema de salud global}

En las últimas décadas, el consumo de drogas ha adquirido una elevada connotación pública, dejando de ser un asunto del ámbito privado, para pasar a formar parte de aquellos problemas sociales sobre los que existe un consenso de que el Estado debiese intervenir (3), ya que el problema de las drogas implica aspectos propios del individuo, como de la familia y la sociedad (4), generando considerables repercusiones en los sistemas asistenciales, así como en la salud de las personas, en los años más productivos de sus vidas (5). En el contexto de la regularización y control de las drogas, la Cannabis sativa, o marihuana, merece consideración especial, por su elevada prevalencia de consumo, la creciente difusión de su uso con fines médicos y la tendencia creciente hacia la despenalización (6). La legalización de la marihuana es un tema que ha concertado el interés de diferentes actores, generando un agudo debate (3). Se ha reportado que la marihuana es la sustancia ilícita más consumida en el mundo $(7,8)$. La Organización de Naciones Unidas (ONU) estima que el consumo mundial oscila entre el 2,9 y el 4,3\% de la población entre 15 y 64 años de edad (7) y que el 4\% de la población 
adulta mundial (162 millones de personas) la consume más de alguna vez durante el año, mientras que el $0,6 \%$ (22 millones) la consume a diario (9), estimándose en la actualidad que unos 20 millones de personas en el mundo son adictas a ella (10). En América Latina la marihuana es ilegal en gran parte de la cadena productiva y Brasil es el país que más consume esta hierba en el subcontinente con un $8,8 \%$ de su población entre 15 y 64 años, seguido de Uruguay con un 8,3\% (9).

\section{Panorama del consumo de marihuana en Chile}

En Chile se ha documentado que la tendencia del consumo de marihuana ha presentado un aumento significativo en los últimos años (11), siendo, el tercer país de Sudamérica en su consumo - un 4,88\% de sus habitantes entre 15 y 64 años son consumidores- (9). La prevalencia de consumo de marihuana en la población entre 12 y 64 años de edad, durante el último año, alcanzó un 6,7\%, cifra menor que las reportadas en los Estados Unidos (12,5\%), Canadá (13,6\%) y Argentina $(7,2 \%)$, pero mayores a la de otros países como Colombia (2,3\%) y Brasil (2,8\%) (12).

En reportes nacionales del año 2013, referentes a su consumo, las cifras obtenidas muestran un aumento de 6,4\% (2008) a un $7,1 \%$ (2012), lo que presenta un quiebre en la tendencia a la baja observada en los estudios (2008 y 2010). Para el grupo de jóvenes (19 a 25 años) se observó un aumento significativo de 5,2 puntos porcentuales (12) y al menos 1 de cada 5 adolescentes que ha usado marihuana en el último año, registra síntomas de dependencia (12). A su vez, los datos advierten prevalencias de marihuana más altas en las regiones de Antofagasta, Coquimbo, Valparaíso y Región Metropolitana (13). No ha sido posible identificar un patrón de comportamiento de consumo de marihuana asociado al nivel socioeconómico y es importante destacar que en Chile, en lo que se refiere a legalizar el uso con fines terapéuticos, ha existido un incremento a favor, de un 59,4\% en el 2010 a un $68,2 \%$ en el año 2014. En lo que respecta a la población escolar chilena la prevalencia de consumo ha aumentado desde $14,8 \%$ en el 2001 a 30,6\% en 2013, siendo mayor en los varones $(31,6 \%)$ que en las mujeres $(29,6 \%)$, aunque no es una marcada diferencia, con prevalencias elevadas ya desde octavo básico $(15,7 \%)$ y que siguen en incremento hasta cuarto medio $(38,9 \%)(13)$.

\section{Posturas en torno a la legalización de la ma- rihuana}

El consumo de marihuana es, en efecto, un problema de salud pública mundial, en respuesta se han generado distintos lineamientos para su enfrentamiento, dentro de los cuales la legalización ha aparecido como una alternativa. En los años 70, Holanda inició su política de tolerancia, permitido la comercialización y consumo en pequeñas cantidades y en los Estados Unidos, desde los años 90, se permite su uso con fines terapéutico en algunos estados y a partir de 2012 dos estados han legalizado el consumo recreativo $(8,14)$. En el contexto latinoamericano, Uruguay fue el primer país de la región en legalizar su uso a partir de diciembre de 2013, bajo una normativa que aún se esta perfilando, aunque no exenta de detractores $(15,16)$, donde "el Estado asume el control y regulación de las actividades de importación y exportación, siembra, cultivo, cosecha, almacenamiento, comercialización y distribución" de la marihuana (17). En Chile, en el año 2015 la Cámara de Diputados aprobó el proyecto de ley que despenaliza el cultivo de marihuana y también su consumo privado, con fines espirituales, medicinales y recreativos. De esta manera, la norma permitirá a los ciudadanos portar cantidades inferiores a diez gramos. Asimismo, se permitirá cultivar un máximo de seis plantas. Igualmente, las personas que necesiten consumir marihuana con fines medicinales deberán tener una 
prescripción médica. La iniciativa modifica el Código Sanitario y la Ley 20.000, que sustituía la Ley 19.366 que sancionaba el Tráfico Ilícito de Estupefacientes y Sustancias Sicotrópicas (18). La iniciativa se encuentra actualmente en discusión en la Comisión de Salud del Senado, lo que ha generado un arduo debate al respecto. Rodríguez (7) señala que ésta es una discusión compleja, ya que se encuentra inmersa en una polarización social, además de ser un tema rodeado de mitos y controversias, cuyos efectos exceden a los individuos que la consumen y abarca de forma transversal dimensiones de la salud, económicas, sociales y políticas (7).

Varios son los países que han aceptado la marihuana como una droga "blanda", diferenciándola de otras más peligrosas y confiándole distintas propiedades terapéuticas (8). Pero hay quienes piensan que su uso debería prohibirse, dadas las interferencias mentales y las alteraciones del comportamiento que produce, ya sea por su consumo esporádico, como también por los daños permanentes a que lleva su consumo crónico (7). Melberg et al. (19) detallan que el consumo de marihuana es un pequeño paso hacia el uso de drogas más fuertes, especialmente en los grupos de consumidores más vulnerables, como lo son los jóvenes. La Drug Enforcement Agency (DEA) (20) caracteriza a la marihuana como una droga adictiva y argumenta que incita el consumo de otras drogas ilícitas, porque se presume que el entorno a que se exponen estas personas aumenta el riesgo. La Organización Mundial de la Salud (OMS) ha identificado el consumo de marihuana como uno de los problemas más graves que aquejan a la sociedad a nivel mundial, a la que se le adjudica una gran potencialidad destructiva, enfatizando los perjuicios de la dependencia tanto física como psicológica que el consumo prolongado produce, afectando tanto al consumidor, a su entorno directo como a la sociedad en su conjunto. El National Institute on Drug Abuse (NIDA) (21) manifiesta que puede tener efectos considerables en la me- moria, la concentración, el apetito, el dolor y la coordinación motriz. Este organismo hace énfasis en el deterioro de la memoria como el principal efecto, ya que el Tetrahidrocarbocannabinol (THC), principal componente de la marihuana (22), altera la manera en que la información es procesada por el hipocampo. Destacan otros efectos asociados, como alteración del estado de ánimo en personas vulnerables y el riesgo de adicción (7). Por otro lado, el National Institute of Health, sostiene que la marihuana podría presentar complicaciones respiratorias al tener componentes similares al tabaco, y que el THC deteriora el sistema inmune (7). De este modo, la potencia de la sustancia, las características específicas de las poblaciones y la conducción después de haberla consumido aumentan el riesgo de accidentes, ya que la marihuana afecta las funciones cognitivas, la memoria y el rendimiento psicomotor (23).

Fischer et al. (23), basándose en una revisión de la evidencia sobre los daños a la salud relacionados con el consumo de marihuana, determinaron que éstos aumentan con la intensidad de su consumo, aunque la curva de riesgo no está bien caracterizada, y además, estarían asociados con un número de factores potencialmente modificables que se relacionan con la frecuencia e inicio temprano del consumo. Es así como varios estudios longitudinales, que estos autores revisaron, sugieren que el inicio temprano del consumo de Cannabis (antes de los 16 años) se asocia con una mayor probabilidad de una variedad de problemas, como el desarrollo de síntomas psicóticos, y, además, que cuanto más temprano en la vida comienza su consumo, más propenso se es de convertirse en consumidor habitual de marihuana (23). Esta es una droga que, en la actualidad, se ha convertido de fácil acceso para los adolescentes que provienen de hogares disfuncionales y está asociada a manifestaciones de corrupción, violencia y al consumo de otras drogas (24). Además, se ha reportado que entre adolescentes y jóvenes consumidores de marihuana es más alta 
la probabilidad de sufrir síndrome de dependencia, verse implicado en accidentes de tránsito, deterioro de la función respiratoria, padecimiento de enfermedades cardiovasculares, así como efectos negativos sobre el desarrollo psicosocial del adolescente y sobre su salud mental, como trastornos psicológicos persistentes (ansiedad y depresión) $(25,26)$.

Referente a sus propiedades terapéuticas, aún no existe consenso de cuáles son sus efectos sobre la salud, esto debido a que es una planta químicamente compleja (7). El Instituto de Medicina de los Estados Unidos indica que sus componentes activos son efectivos para el tratamiento del dolor, náuseas (ocasionadas por quimioterapia), anorexia por SIDA, entre otros; pero que estos efectos se deben evaluar clínica y rigurosamente. Instituciones Internacionales de Salud como: American Public Health Association, Health Canadá y Federation of American Scientists apoyan el acceso legal y medicinal para pacientes cuya condición física lo requiera, previa evaluación médica (7). En el contexto nacional, Mönckeberg (8) afirma que los productos derivados de la marihuana pueden llegar a ser fármacos muy útiles en la medicina moderna, y agrega que la industria farmacéutica puede utilizarla como un producto farmacológico, por lo que no se requiere liberalizar su consumo. Por otra parte, se considera que la marihuana es un estupefaciente peligroso y que se torna un tema complejo al considerar que Chile no tiene una cultura que refleje un perfil basado en el autocontrol, basándose en que cerca del $80 \%$ no es capaz de entender lo que lee, lo que significa que sólo un $20 \%$ tendría un criterio absolutamente formado al respecto (27). Criterio que puede ser influenciado por los medios de comunicación que actualmente tienden a minimizar las consecuencias del uso de la planta, principalmente en los adolescentes, quienes están recién en un proceso de maduración, sin una clara firmeza valórica y con una toma de decisiones influida muchas veces por sus pares. Es así como la percepción de riesgo de consumir marihuana en la pobla- ción escolar chilena ha bajado notoriamente desde un 51,3\% (2001) a un 21,1\% (2013), incluso ha caído la desaprobación parental de su uso desde un 82,3\% (2011) a un 73,9\% (2013) (13).

Por su parte Volkow (28), directora del National Institute on Drug Abuse (NIDA), también se ha referido a la disminución social en la percepción del riesgo, indicando que va de la mano con el aumento en el consumo y que existe un escepticismo creciente de los jóvenes sobre los peligros de la marihuana, a pesar que la evidencia señala que su consumo durante la adolescencia tiene el potencial de cambiar negativamente el curso de vida de los jóvenes, impidiendo que desarrollen todo su potencial, afectando negativamente su trayectoria de vida debido a una suma de factores que incluyen alteraciones en el desarrollo cognitivo y social (28). En este sentido, un estudio realizado el 2014 por el Instituto Nacional de la Juventud (INJUV) (29) entre jóvenes de 15 a 29 años, reveló que un 38\% de los jóvenes consideran que la marihuana es poco o nada dañina para la salud, considerándola menos dañina que el alcohol o el tabaco. Además, el 63\% de la juventud esta de acuerdo o muy de acuerdo con la despenalización de la marihuana como estrategia de control de consumo y ante una posible despenalización, el $43 \%$ de las y los jóvenes señala que su consumo aumentaría, mientras que un $40 \%$ dice que se mantendría. Un $60 \%$ señala que como sociedad estamos preparados para su despenalización y el $81 \%$ de los entrevistados considera que hoy es más fácil conseguir marihuana que hace 10 años (29).

\section{Perspectivas de enfermería en Chile respec- to a la legalización de la marihuana}

En Chile, enfermería ha aparecido distante de la discusión específica sobre la legalización de la marihuana, no se han encontrado posturas emanadas desde asociaciones gremiales ni colegio profesional. Sí existen antecedentes de trabajo conjunto, entre los años 2005 y 
2008, de la Asociación Chilena de Escuelas de Enfermería (ACHIEEN), la Comisión Interamericana para el Control del Abuso de Drogas (CICAD) y el Consejo Nacional para el Control de Estupefacientes (CONACE), con el fin de formar a los profesionales de enfermería en el área de reducción de la demanda de drogas. Desde ese proyecto, la ACHIEEN tomó la decisión de hacerse parte de apoyar la tarea de reducir la demanda de drogas en Chile, con la convicción de que las enfermeras son agentes de salud fundamentales que pueden apoyar a producir el cambio y la transformación en salud de las personas, familias y comunidades (30).

El problema del uso y abuso de drogas requiere adquirir primacía en la preocupación y el desarrollo profesional, pero no sólo interviniendo cuando el problema ya está presente sino que las enfermeras/os sean capaces de prevenirlo a tiempo ya que, a pesar de los esfuerzos realizados, estos fenómenos van en alza. En este ámbito Malvárez (31) indica que las transiciones demográficas y epidemiológicas, unidas a las consecuencias de la globalización presentan un panorama cambiante que exige de la enfermería un franco reposicionamiento en relación a su tradición profesional. Como señala Paravic (32), "la globalización exige a enfermería conocer los valores, creencias, costumbres, necesidades, gustos, idiomas de los diferentes pueblos", ante lo cual se hace indispensable, formar profesionales de enfermería, altamente calificados y preparados, con capacidades creativas y de innovación, flexibles pero también rigurosos para hacer frente a los retos de este mundo global. Por su parte, López (33) expone que la globalización "impacta en las funciones de enfermería" e indica que debería haber una orientación al aumento en la calidad y estilos de vida saludables, actuar sobre los factores de riesgo e identificar y fortalecer los factores protectores. Ello adquiere más valor en el tema de las drogas, ya que como expone la OMS, se ha demostrado que los programas de tratamiento para el consumo de drogas reducen los costos sociales y de salud en una escala mucho mayor que el costo mismo del tratamiento (12). En los últimos 5 años se ha desarrollado evidencia a favor de la prevención del abuso de drogas, la que debe tener un enfoque centrado en el desarrollo de habilidades sociales, comunicativas, incorporación de la familia, promoción de una mayor conciencia y relevancia de la percepción de los efectos perjudiciales que resultan de su uso, creación de redes sociales, enseñanza de habilidades de resistencia social, por sobre aquellos ineficaces enfoques de prevención tradicionales que se basan en información relativa a la enseñanza de las consecuencias adversas de la droga y el abuso (34). Wright (35) señala que la "reducción de la demanda de drogas es un área nueva de trabajo que se abre para la profesión de enfermería, la cual puede y debe tener liderazgo y aplicar los conocimientos científicos y técnicos disponibles", para tener un rol protagónico tanto en la prevención como en el cuidado durante el tratamiento y la rehabilitación. En este sentido, bajo la perspectiva de la salud internacional existen propuestas novedosas para el trabajo con el fenómeno de las drogas. El Modelo Crítico-Holístico de Salud Internacional aplicado al estudio del fenómeno de las drogas y de la violencia podrá abrir nuevos espacios de investigación de enfermería sobre drogas y violencia (35).

Enfermería está preparada para desarrollar actividades enfocadas a prevenir el uso de drogas, interviniendo sobre los factores de riesgo y promoviendo la integración en la familia y en los demás segmentos sociales. Puede actuar en el fomento de la salud, fortaleciendo los factores protectores, con miras a la mejoría de la autoestima y la auto-eficacia, y desarrollar estrategias para el mantenimiento de la salud. Con programas de prevención principalmente en los adolescentes, que han demostrado eficacia a largo plazo y retraso en el involucramiento de este grupo en conductas de alto riesgo. En relación con el tratamiento de la dependencia de 
las drogas, el profesional de enfermería debe responsabilizarse del cuidado de enfermería y participar en el diseño y ejecución del plan de cuidado, establecido en conjunto con el equipo de salud y el paciente. Pero el inconveniente que se vislumbra en este aspecto son los recursos con que se cuenta para responder al problema de las adicciones y sus implicaciones sociales y sanitarias. Según datos de la OEA (6), la tasa de enfermeras (con especialidad en psiquiatría y generales) por habitantes, a nivel de las Américas, es de 3,92 por 1.000 habitantes, un $32 \%$ más baja que la tasa mundial. Específicamente en Chile la tasa de enfermeras(os) que trabajan en salud mental es de 1,70 por 100.000 habitantes, un número muy reducido para hacer frente a este fenómeno que va en aumento. Por lo que se torna necesario la formación de enfermeras en esta área.

Desde otra perspectiva, enfermería es una de las profesiones del área de la salud que viene ampliando sus espacios en el debate de cuestiones sociales, económicas, políticas, de educación y de salud (36). A pesar de esto no resulta fácil posicionarse en el tema de la legalización de la marihuana ya que involucra variadas aristas; por un lado es un fenómeno protagonizado por seres humanos donde se mezclan los principios, valores y creencias de la persona bajo el cuidado de enfermería, como las del propio profesional, tiene una relevancia epidemiológica a nivel local, nacional e internacional y, por otro, abarca factores muy diversos: económicos, culturales, sociales, políticos, sanitarios, religiosos, entre otros (37). Pero es importante que enfermería avance en este tema, considerando que el desafío de cuidar a las comunidades en un mundo global involucra un cambio de paradigma de la noción de cuidado, que supone un cambio de problemas, abordajes, tecnologías, escenarios diferentes y prácticas distintas (31). En otro sentido, y considerando las creencias personales de los sujetos en torno al uso de la marihuana, emerge el Cuidado Cultural de Enfermería, cuando los enfermeros/as conocen los valores culturales, creencias y modo de vida de los individuos, el cuidado que se brinda es mucho más provechoso e integral (38). Sumado a lo anterior no pueden estar fuera del análisis los aspectos bioéticos implicados ya que como lo expone Pérez (37), la reflexión bioética aporta una perspectiva complementaria a la terapéutica, además que en las relaciones humanas no existen actos éticamente neutros. Es así como al considerar el principio de justicia debe cuidarse el dar a cada sujeto lo que necesita sin discriminarle sanitariamente por no alcanzar las expectativas sociales y, desde el principio de autonomía, respetar su individualidad y su proyecto vital, abordándolo como un individuo social con sus derechos y responsabilidades, permitiendo su integración y su normalización (37). Los principios de no maleficencia y beneficencia hacen referencia al deber de no dañar a la persona y hacer el bien a quien lo pide $(37,39)$.

\section{CONSIDERACIONES FINALES}

Ante todo lo expuesto, el análisis del tema de la legalización de la marihuana desde la perspectiva de enfermería no es algo sencillo, pero se considera que debería involucrar a lo menos cinco criterios: identificación y evaluación de la mejor evidencia científica disponible; conocer las posturas de los principales actores en el tema; el cambio de paradigma de la noción de cuidado con un abordaje más intersectorial y abierto a practicas distintivas; los valores, creencias, estilos de vida de las personas bajo nuestro cuidado desde su propia óptica y finalmente los principios de la bioética atinentes.

No cabe duda que el análisis de los conflictos bioéticos relativos a la legalización van mucho más allá de lo que se ha comentado, pero este ensayo no pretende otra cosa que un llamado a la reflexión, con la finalidad de consensuar posturas al respecto. El posicio- 
namiento de enfermería frente al tema de la legalización de la marihuana es dilemático ya que, estamos frente a una persona a la que se le debe respetar el proyecto de vida y la idea del bien de cada cual y como afirma Gracia (40), esto es independiente de que no estemos de acuerdo con ellos y finalmente que la sociedad puede y debe imponer límites a la libertad de los individuos a fin de proteger el bien común y la justicia.

Reconociendo todo lo anterior, las autoras consideran que es necesario avanzar hacia la regulación de la marihuana en lo que dice relación con sus aplicaciones terapéuticas científicamente fundadas como producto farmacológico y que el enfoque en común que debiera adoptar enfermería y que adquiere mayor relevancia es la prevención de su uso, actividad en la cual enfermería puede y debe tener liderazgo (41) trabajando proactivamente a nivel comunitario y específicamente en los establecimientos educacionales.

La evidencia del uso terapéutico de la marihuana está en pleno desarrollo y en la actualidad demuestra resultados diversos. Es necesario profundizar aún más utilizando metodologías científicas adecuadas. El debate de la legalización de la marihuana es un tema que se viene dando desde hace décadas con una inclinación más en contra que a favor por la sólida evidencia empírica de los daños a la salud que produce y los retrocesos que han experimentado algunos países que ya la han legalizado. En Chile este debate es más reciente, pero ya ha generado diferentes posturas. Las enfermeras y enfermeros, siendo el grupo más amplio de profesionales que proporcionan servicios de salud (42), no pueden ni deben retraerse de esta discusión, tienen una gran responsabilidad en contribuir a mejorar la salud de la población y deben comenzar a discutir el tema.

El llegar a un posicionamiento requiere de un profundo consenso y reflexión. Pero el enfoque en común de enfermería debe radicar en la prevención del uso de la marihuana en etapas escolares tempranas.

\section{REFERENCIAS}

1. Marimón N, Martínez E. Globalización, Salud y Solidaridad: una visión diferente. Rev Cub Salud Pública [Internet]. 2009 Mar [citado 11 Abr 2014]; 35(1). Disponible en: http://www.scielosp.org/scielo. php?script $=$ sci_arttext\&pid $=$ S0864 $34662009000100014 \& \operatorname{lng}=e n$. http://dx.doi.org/10.1590/S08643466200900010001

2. García M., Ramírez A. La educación de enfermería y las adicciones. Una revisión de la literatura. Rev. enferm. Inst. Mex. Seguro Soc. 2010; 18(1): 35-42.

3. Hurtado P. Determinantes del consumo de marihuana en Chile. Análisis de los datos de autorreporte. Estudios públicos [Internet]. 2006 [citado 11 Abr 2014]; 102: 148-177. Disponible en : http:// www.cepchile.cl/cep/site/artic/20160304/ asocfile/20160304094019/r102_hurtado_ marihuana.pdf

4. Ruiz J. Manual Básico de Adicciones. La Plata: La comuna ediciones; 2003. 104 p.

5. Organización Panamericana de la Salud (OPS). Epidemiología del uso de drogas en América Latina y el Caribe: un enfoque de salud pública [Internet]. Washington, D. C.: OPS; 2009 [citado 11 Abr 2014]. 36 p. Disponible en : http://www. paho.org/hq/dmdocuments/2009/epidemiologia_drogas_web.pdf

6. Insulza J. El problema de las Drogas en las Américas: Capítulo 2: Drogas y Salud Pública [Internet]. Washington, D. C.: Organización de los Estados Americanos; 2013 [citado 15 Abr 2015]. 79 p. Disponible en: http://www.cicad.oas.org/drogas/ elinforme/informeDrogas2013/drugsPublicHealth_ESP.pdf

7. Rodríguez ME. Desafíos que enfrenta el debate y la propuesta de la legalización del Cannabis. Trabajos de Investigación en Políticas Públicas (TIPS) [Internet]. Santiago de Chile: Departamento de Eco- 
nomía, Universidad de Chile; 2013 [citado 11 Abr 2014]; 17: 1-17. Disponible en: http://new.econ.uchile.cl/uploads/ publicacion/eb05aa230306d9187a71b345ce8ea11e6f511832.pdf

8. Mönckeberg F. Los Pro y Contra de la Legalización de la Marihuana. Rev Chil Pediatr 2014; 85 (2): 229-237.

9. United Nations on Drug and Crime (UNODC). World Drug Report 2013 [Internet]. Vienna: United Nations; 2013 [citado 14 abr 2014]. 115 p. Disponible en: http://www.unodc.org/documents/ lpo-brazil//Topics_drugs/WDR/2013/ World_Drug_Report_2013.pdf

10. United Nations on Drug and Crime (UNODC). World Drug Report 2014 [Internet]. Vienna: United Nations; 2014 [citado 14 Abr 2014]. 115 p. Disponible en https://www.unodc.org/documents/ wdr2014/World_Drug_Report_2014_ web.pdf

11. Observatorio Chileno de Drogas. Boletín $\mathrm{N}^{\circ}$ 12: Consumo de Marihuana en Población Escolar: Análisis de prevalencia e incidencia. [Internet]. Santiago; 2015 [citado 11 Abr 2014]; 1-5. Disponible en: http://www.senda.gob.cl/media/boletines/Boletin\%2012\%20Consumo\%20de\%20Marihuana\%20en\%20 Poblaci\%C3\%B3n\%20Escolar\%20 An\%C3\%A1lisis\%20de\%20prevalencia\%20e\%20incidencia.pdf

12. Ministerio de Salud (MINSAL). Guía Clínica AUGE "Consumo perjudicial y Dependencia de alcohol y otras drogas en personas menores de 20 años" [Internet]. Santiago de Chile: MINSAL; 2013 [citado 15 abr 2014]. 92 p Disponible en: file:///E:/Guia $\% 20$ Clinica $\% 20$ Auge $\% 20$ 2013.pdf

13. Observatorio Chileno de Drogas. Servicio Nacional para la Prevención y Rehabilitación del Consumo de Drogas y Alcohol (SENDA). Décimo estudio nacional de drogas en población general de Chile, 2012 [Internet]. Santiago de Chile:
SENDA; 2013 [citado 16 May 2014]. 174 p. Disponible en http://www.senda.gob. cl/media/estudios/PG/Decimo\%20Estudio\%20de\%20Drogas\%20Pob\%20General\%202012.pdf

14. Campero JC, Barrancos H., Vargas R., Vergara E., Brombacher D., Stöver H., et al. De la represión a la regulación: propuestas para reformar las políticas contra las drogas. Bogotá: Friedrich Ebert Stiftung (FES); 2013. 397 p.

15. Pettitt-Schieber B. El "Amanecer Verde": La legalización de la Marihuana en Uruguay en el Contexto del Movimiento Regional Contra la Prohibición [Internet]. Buenos Aires/Montevideo: Independent Study Project (ISP) Collection. Paper 1399; 2012. [citado 11 Abr 2014]. 44 p. Disponible en: http://digitalcollections. sit.edu/isp_collection/1399

16. Bidegain G. Uruguay: ¿El año Bisagra? Rev. cienc. polít. (Santiago) [Internet]. 2013 [citado 16 Abr 2014]; 33(1):351-374. Disponible en: http://www.scielo.cl/scielo.php?script=sci_arttext\&pid=S0718090X2013000100017

17. Klein D, Romo R. Uruguay decide este martes si aprueba la ley que legaliza la marihuana [Internet]. CNN en español; 2013 Dic 9 [citado 16 ago 2016]. Disponible en: http://cnnespanol.cnn. com/2013/12/09/uruguay-decide-estemartes-si-aprueba-la-ley-que-legalizala-marihuana/

18. Cámara de Diputados de Chile. Cannabis: Diputados aprueban en general proyecto que legaliza autoconsumo y despenaliza uso medicinal [Internet]. Noticias Cámara de Diputados de Chile; 2015 Jul 07 [citado 16 ago 2016]. Disponible en: https://www.camara.cl/prensa/noticias_ detalle.aspx?prmid $=126715$

19. Melberg HO, Bretteville-Jensen A, Jones A. Is Cannabis a Gateway to Hard Drugs? Empirical Economics. 2010; 38(3): 583603.

20. U. S. Department of Justice/ Drug En- 
forcement Administration. Drug of Abuse [Internet]. Washington D. C.: Drug Enforcement Administration/U. S. Department of Justice; 2011. [citado 16 ago 2016]. Disponible en: https://www.dea. gov/pr/multimedia-library/publications/ drug_of_abuse.pdf

21. National Institutes of Health (NIH)/National Institute on Drug Abuse. Marijuana Brief. Description [Internet]. Bethesda, Maryland: NIH; 2014. [citado 16 ago 2016] Marijuana Brief. Description. Disponible en: https://www.drugabuse.gov/ drugs-abuse/marijuana

22. Hardisson A, Expósito C, Rubio C, Pozuelo MR. Nuevas perspectivas terapéuticas de los compuestos cannabinólicos. Rev. toxicol. 2002; 19: 89-92.

23. Fischer B, Jeffries V, Hall W, Room R, Goldner E, Rehm J. Lower Risk Cannabis Use Guidelines for Canada (LRCUG): A Narrative Review of Evidence and Recommendations. Can J Public Health. 2011; 102(5): 324-27.

24. Bueno L, Guerrero J, Javier R, Tam E. Prevalencia de consumo de marihuana en estudiantes de secundaria de instituciones educativas estatales de Ventanilla. Rev enferm Herediana. 2015; 8(1): 17-23.

25. Hall W, Degenhardt L. Adverse health effects of non-medical cannabis use. The Lancet. 2009; 347(9698): 1383-1391.

26. Swift W, Coffey C, Carlin JB, Degenhardt L, Patton GC. Adolescent cannabis users at 24 years: trajectories to regular weekly use and dependence in young adulthood. Addiction. 2008; 103(8): 1361-1370.

27. Rojas C. Experto en adicciones por marihuana: "Chile no está preparado culturalmente para la despenalización" [Internet]. Diario La Tercera; 2013 May 20 [citado 16 ago 2016]. Disponible en: http://www.latercera.com/noticia/ nacional/2013/05/680-524325-9experto-en-adicciones-por-marihuanachile-no-esta-preparado-culturalmentepara.shtml
28. National Institute on Drug Abuse (NIDA). Messages From the Director. Desafiando los mitos sobre la marihuana [Internet]. NIH; 2013 Feb [citado 2016-08-16]. Disponible en: http://www.drugabuse.gov/ es/about-nida/directors-page/messagesdirector/2013/02/desafiando-los-mitossobre-la-marihuana

29. Ministerio de Desarrollo Social Gobierno de Chile/INJUV. Drogas y Legalización de marihuana: Visión de los jóvenes chilenos [Internet]. Santiago de Chile: INJUV; 2014. [citado 16 agosto 2016]. Disponible en: http: http://www.injuv.gob.cl/portal/rt-blog/numero-12-diciembre-2014/ drogas-y-legalizacion-de-la-marihuanavisiones-de-los-jovenes-chilenos/

30. ACHIEEN/CONACE/CICAD-OEA. Formando enfermeras(os) en el fenómeno de las adicciones. Proyecto ACHIIEN/ CONACE/CICAD 2005-2008. Santiago de Chile: ACHIEEN-CONACE, Gobierno de Chile; 2009. $124 \mathrm{p}$.

31. Malvárez S. El reto de cuidar en un mundo globalizado. Texto Contexto Enferm. 2007; (3): 520-530.

32. Paravic T. Enfermería y Globalización. Cienc. enferm. 2010; XVI(1): 9-15.

33. López M. Impacto de la Globalización en las Funciones de Enfermería. Acalan [Internet]. 2003 [citado 11 Abr 2014]; 28. Disponible en: http://www.unacar.mx/ contenido/difusion/acalan28pdf/acalan28.pdf

34. Pentz MA. Costs, benefits, and cost-effectiveness of comprehensive drug abuse prevention. In: Bukoski WJ, and Evans RI, eds. Cost-Benefit/Cost-Effectiveness Research of Drug Abuse Prevention: Implications for Programming and Policy. NIDA Research Monograph No 176 . Rockville: National Institute on Drug Abuse; 1998. p. 111-129.

35. Wright MGM. La Contribución de la Enfermería frente al Fenómeno de las Drogas y la Violencia en América Latina: Un Proceso de Construcción. Cienc. en- 
ferm. 2002; VIII(2): 9-19.

36. Wright MGM. El Análisis crítico-holístico de los Programas de Postgrado de Enfermería frente a los Desafíos de la reducción de la demanda de drogas en América Latina. En: Wright MGM, Chisman AMCG, Padilha MICS, Gelbcke FL, Pires DEP, Radunz V, et al, editores. La Situación de los Programas de Postgrado de Enfermería en Nueve Países de América Latina Frente a los Desafíos de la reducción de la demanda de drogas. Florianópolis (SC): OEA / CICAD / UFSC; 2003. p. 15-22.

37. Pérez M. Aspectos bioéticos de la asistencia al drogodependiente. Adicciones. 2000; 12(4): 515/526.

38. Castillo J. El Cuidado Cultural de Enfermería. Necesidad y Relevancia. Rev haban cienc méd [Internet]. 2008 JulSep [citado 16 abr 2014]; 7(3). Disponible en: http://scielo.sld.cu/scielo. php? script $=$ sci_arttext\&pid $=$ S1729-
519X2008000300003

39. Gárciga O, Alburquerque F. Las conductas adictivas: análisis crítico y propuestas para su prevención. Rev. Hosp. Psiquiátrico de La Habana. 2012; 9(2).

40. Gracia D. Por una cultura de la responsabilidad en el tema de las drogas. En: Gracia D, ed. Las Drogas a debate: Ética y Programas de Sustitución, Fundación Ciencias de la Salud. Madrid: Ediciones Doce Calles SL; 1999. p. 242-263.

41. Wright MGM, Gliksman L, Khenti A, Furegato ARF. Investigación sobre el Fenómeno de las Drogas bajo el abordaje de los Estudios Multicéntricos en América Latina y Caribe. Rev Lat Am Enfermagem. 2009; 17(7): 59-61.

42. Pereira RPG, Cardoso MJSP, Martins MACS. Atitudes e barreiras à prática de enfermagem baseada na evidência em contexto comunitário. Rev. Enf. Ref. 2012; ser III(7): 55-62. 